\title{
Genetic association of interleukin-21 polymorphisms with systemic lupus erythematosus
}

\author{
A H Sawalha, ${ }^{1,2,3}$ K M Kaufman, ${ }^{1,2,3} \mathrm{~J} \mathrm{~A} \mathrm{Kelly,}{ }^{3}$ A J Adler, ${ }^{3}{ }^{\text {T Aberle, }}{ }^{3}$ J Kilpatrick, ${ }^{3}$ \\ E K Wakeland, ${ }^{4}$ O-Z Li, ${ }^{4}$ A E Wandstrat, ${ }^{4}$ D R Karp, ${ }^{5}$ J A James, ${ }^{3}$ J T Merrill, ${ }^{2,3}$ P Lipsky, ${ }^{6}$ \\ J B Harley ${ }^{1,2,3}$
}

${ }^{1}$ US Department of Veterans Affairs Medical Center,

Oklahoma City, Oklahoma, USA;

${ }^{2}$ Department of Medicine,

University of Oklahoma Health

Sciences Center, Oklahoma City,

Oklahoma, USA; ${ }^{3}$ Arthritis and

Immunology Program, Oklahoma

Medical Research Foundation,

Oklahoma City, Oklahoma, USA;

${ }^{4}$ Center for Immunology,

University of Texas

Southwestern Medical Center,

Dallas, Texas, USA;

${ }^{5}$ Department of Medicine,

University of Texas

Southwestern Medical Center,

Dallas, Texas, USA;

${ }^{6}$ Autoimmunity Branch, National Institute of Arthritis and

Musculoskeletal and Skin

Diseases, Bethesda, Maryland,

USA

Correspondence to:

Amr H Sawalha, 825 N.E. 13th

Street, MS\#24, Oklahoma City,

Oklahoma 73104, USA; amr-

sawalha@omrf.ouhsc.edu

Accepted 12 August 2007

Published Online First

24 August 2007

\begin{abstract}
Objective: The aetiology of systemic lupus erythematosus (SLE) is incompletely understood. Both genetic and environmental factors are implicated in the pathogenesis of the disease. Herein, we describe genetic association between SLE and polymorphisms in the interleukin (IL)-21 gene. The reported effect of IL-21 on B-cell differentiation into plasma cells and its effect on dendritic cell maturation and T-cell responses make IL-21 an attractive candidate gene for SLE.
\end{abstract}

Methods: Three single nucleotide polymorphisms (SNPs) in the IL-21 gene were genotyped in a total of 2636 individuals (1318 cases and 1318 controls matched for age, sex and race). Population-based case-control association analyses were performed.

Results: We found a genetic association with SLE and two SNPs located within the IL-21 gene (rs907715: $\chi^{2}=11.55, p<0.001 ;$ rs2221903: $\chi^{2}=5.49$,

$p=0.019$ ). Furthermore, genotypes homozygous for the risk alleles were more frequent than genotypes homozygous for the non-risk alleles in European-American patients as compared to controls (rs907715 (GG versus $\mathrm{AA}$ ): odds ratio $(\mathrm{OR})=1.66, \mathrm{p}=0.0049$; rs2221903 (GG versus $A A): O R=1.60, p=0.025$ ).

Conclusion: Our findings indicate that IL-21 polymorphism is a candidate association with SLE. The functional effects of this association, when revealed, might improve our understanding of the disease and provide new therapeutic targets.

Systemic lupus erythematosus (SLE) is a chronic autoimmune disease characterised by the production of autoantibodies directed against a host of nuclear components. The disease is relapsing and affects multiple organ systems including the skin, kidneys, lungs, heart and the central nervous system. The aetiology of SLE is incompletely understood, but is likely multifactorial. Both genetic and environmental factors are thought to play a role in the pathogenesis of SLE. Familial aggregation of the disease, a higher reported concordance rate in monozygotic compared to dizygotic twins, and the multiple genetic linkages and associations discovered all suggest an important genetic component contributing to the aetiology of SLE. ${ }^{1}$ In addition, a large body of evidence accumulated over the past 20 or so years suggests a role for abnormal DNA methylation in the pathogenesis of drug-induced and idiopathic SLE. ${ }^{2}$

SLE is heterogeneous in its presentation and manifestations. Therefore, two patients can be diagnosed with SLE, despite sharing no clinical manifestations. The one unifying finding among virtually all SLE patients is the presence of autoantibodies directed against nuclear antigens. ${ }^{3}$ Indeed, autoantibodies are present in the sera of SLE patients years before the development of clinical disease. ${ }^{4}$ Many mechanisms through which the various autoantibodies mediate pathogenecity are areas of active investigation.

SLE is characterised by the presence of an autoreactive CD4+ $\mathrm{T}$ cell subset and increased antibody production. $\mathrm{CD} 4+\mathrm{T}$ cells that are made autoreactive in vitro, for example by inhibiting DNA methylation, are capable of stimulating autologous B cells, resulting in increased antibody production. ${ }^{5}$ This suggests an important role for T-cell dependent B-cell activation in SLE. Interleukin (IL)-21 is a newly-discovered cytokine produced by activated $\mathrm{CD} 4+\mathrm{T}$ cells that promotes B-cell differentiation, ${ }^{6}$ and is therefore a good candidate gene for this disease. Using a population-based case-control approach, we found a genetic association of IL-21 and SLE.

\section{METHODS}

\section{Patients and controls}

SLE patients and healthy controls were enrolled in the lupus genetics studies at the Oklahoma Medical Research Foundation. A total of 1318 independent SLE patients and 1318 unrelated healthy controls matched for age, sex and race were evaluated. Our SLE patient population included 1164 female and 154 male SLE patients. Stratified by race, our patient population consisted of 644 European-Americans, 366 AfricanAmericans, 117 Hispanic Americans, 108 Gullah (African-Americans who live in the low country region of South Carolina and Georgia) patients, and 83 patients of other ethnicities. All SLE patients studied fulfilled at least four of the American College of Rheumatology (ACR) classification criteria for SLE.? No two SLE patients or controls came from the same family in order to avoid intra-familial correlation. Medical data were abstracted from medical records as well as from participant questionnaires and interviews. The presence or absence of SLE clinical criteria was verified by either a physician or a physician assistant in our group, and the finding of objective documentation was required to consider the presence of clinical criteria used for classification.

\section{Genotyping}

Upon obtaining informed consent, genomic DNA was isolated from peripheral blood mononuclear 


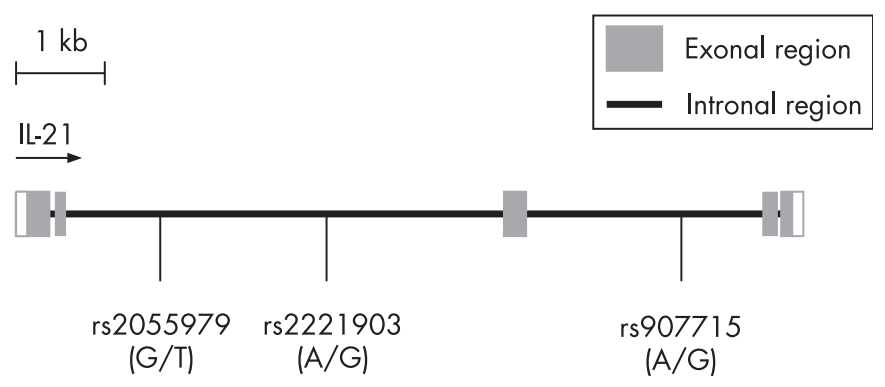

Figure 1 Interleukin (IL)-21 gene region demonstrating the single nucleotide polymorphisms (SNPs) genotyped in this study. The SNPs rs2055979 (G/T) and rs2221903 (A/G) are located within the second intron, rs907715 (A/G) is located in the third intron of IL-21.

cells, lymphoblastoid cell lines, or buccal cell swabs as previously described. ${ }^{8}$ Three single nucleotide polymorphisms (SNPs) in the IL-21 gene were genotyped in the 2636 samples (fig 1). These SNPs were selected to cover a large area in the IL21 gene. The SNPs selected were validated polymorphisms, and have a minor allele frequency of more than 10\%. Genotyping was performed using the Golden Gate SNP assays on the Illumina BeadStation 500GX system (San Diego, California, USA) at the University of Texas Southwestern Microarray Core Facility (Dallas, Texas, USA). Genotyping success rate was $100 \%$ for all of the three SNPs genotyped.

\section{Statistical analysis}

Population-based case-control association designs were employed. The frequencies of the SNP alleles were compared in patients and controls using standard Pearson $\chi^{2}$ tests. $p$ Values of $<0.05$ are reported without correction for multiple comparisons. Odds ratios with $95 \%$ CIs were calculated under the assumption of normality. Fisher's exact method was used to test for deviation from Hardy-Weinberg equilibrium in the genotyped SNPs (http://ihg.gsf.de/cgi-bin/hw/hwa1.pl).
Haplotype analysis was performed using the Haploview program version $3.32 .^{9}$

Conditional logistic regression was used to compute adjusted odds ratios for any associations with the 11 ACR criteria in the European-American patients who were homozygous for the risk alleles in the associated SNPs compared to patients homozygous for the non-risk alleles. Independent variables included malar rash, discoid rash, photosensitivity, oral ulcers, arthritis, pericarditis, pleuritis, proteinuria, cellular casts, seizures, psychosis, haemolytic anaemia, leucopoenia, lymphopenia, and thrombocytopenia as defined. ${ }^{7}$ All of the variables were dichotomous.

\section{RESULTS}

We genotyped three SNPs located within the IL-21 gene in 1318 SLE patients and 1318 matched controls (fig 1). We found genetic association between SLE and two of the three IL-21 SNPs genotyped. The SNP rs907715 had a minor allele frequency of $35 \%$ in SLE patients as compared to $39 \%$ in healthy controls $\left(\chi^{2}=11.55, p<0.001\right)$. The other associated SNP, rs2221903, had a minor allele frequency of $21 \%$ in SLE patients and $19 \%$ in matched controls $\left(\chi^{2}=5.49, p=0.019\right)$. Both associated SNPs are located within intronal regions of the IL-21 gene. By contrast, the allele frequencies of rs2055979 were similar between patients and controls $(20 \%$ versus $21 \%$ respectively, $\chi^{2}=0.66, p=0.42$ ).

Upon stratification based on ethnicity, rs907715 and rs2221903 were associated with SLE in European-Americans (table 1). The odds for carrying the risk allele at rs 907715 were 1.29 greater in European-American SLE patients compared to matched controls $(95 \% \mathrm{CI}=1.10-1.52 ; \mathrm{p}=0.002)$. Odds for carrying the risk allele at rs2221903 were 1.19 greater in European-American patients compared to controls $(95 \%$ $C I=1.01-1.41 ; p=0.044)$. Similarly, rs2221903 showed association in African-American patients (odds ratio $=1.63 ; 95 \%$ $\mathrm{CI}=1.10-2.41 ; \mathrm{p}=0.014)$. No association was detected in the Hispanic or Gullah populations.

Table 1 Allele and genotype frequencies of rs907715 (A/G) and rs2221903 (A/G) in European-American and African-American systemic lupus erythematosus (SLE) patients and controls

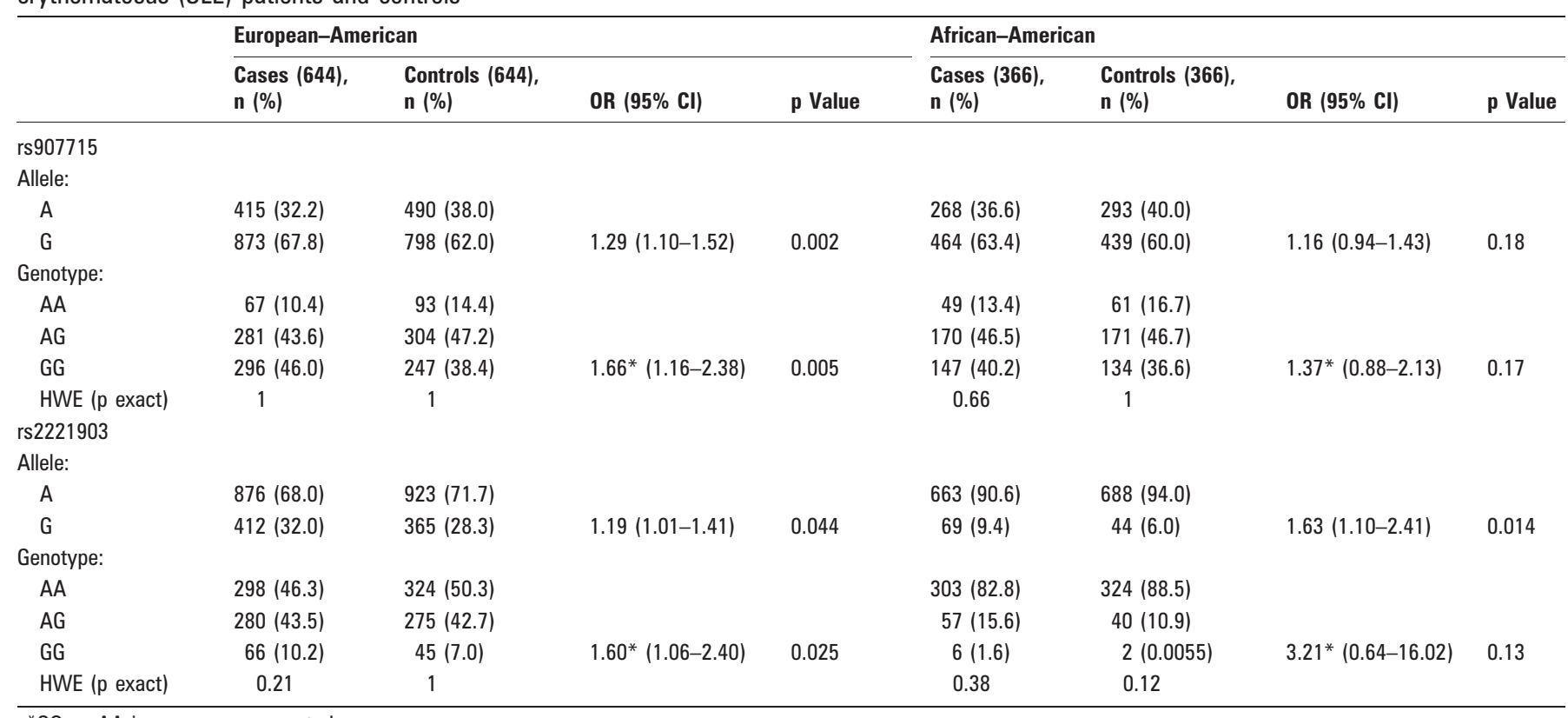


Table 2 Haplotype frequencies of rs907715 (A/G) and rs2221903 (A/G) in European-American systemic lupus erythematosus (SLE) patients and controls

\begin{tabular}{llllll}
\hline Haplotype & & & & & \\
\cline { 1 - 2 } rs907715 & rs2221903 & Cases (\%) & Controls (\%) & $\chi^{2}$ & p Value \\
\hline A & A & 0.323 & 0.380 & 9.41 & 0.0022 \\
G & A & 0.358 & 0.337 & 1.31 & 0.25 \\
G & G & 0.319 & 0.283 & 4.01 & 0.045 \\
\hline
\end{tabular}

We compared the relative frequencies of homozygotes for the associated alleles to the homozygotes for the non-associated alleles for rs907715 and rs2221903 in the cases and controls. Interestingly, the frequency of the rs907715 (GG) genotype was $46.0 \%$ in European-American SLE patients compared to only $38.4 \%$ in matched controls $(\mathrm{OR}=1.66(95 \% \mathrm{CI}=1.16-2.38)$, $\mathrm{p}=0.0049$ ). Likewise, the rs2221903 (GG) genotype was present in $10.2 \%$ of European-American SLE patients compared to $7.0 \%$ in controls $(\mathrm{OR}=1.60 \quad(95 \% \quad \mathrm{CI}=1.06-2.40), \quad \mathrm{p}=0.025)$ (table 1).

Haplotype analysis of rs907715 and rs2221903 in EuropeanAmerican SLE patients identified three haplotypes. The frequencies of the AA and GG haplotypes were significantly different between SLE patients and controls (AA: $\chi^{2}=9.41$, $p=0.0022$; GG: $\chi^{2}=4.01, p=0.045$ ), with the GG haplotype demonstrating risk in our samples and the AA haplotype demonstrating a protective effect in our samples (table 2).

To determine if the SLE-associated IL-21 SNPs described herein are associated with a particular clinical manifestation in SLE patients, we determined the frequency of the various SLE manifestations in European-American patients that were homozygous for the risk alleles (GG/GG) compared to patients homozygous for the non-risk alleles (AA/AA) at rs907715 and rs2221903. Surprisingly, we found that European-American SLE patients with the GG/GG genotype were $\sim 8$ times less likely to suffer from CNS lupus involvement, particularly seizures, compared the SLE patients who carried the AA/AA genotypes. Only 1 of 53 patients with the GG/GG genotype had seizures compared to 8 of 55 patients with the AA/AA genotype $(1.9 \%$ versus $14.6 \%, \mathrm{OR}=0.11,95 \% \mathrm{CI}=0.01-0.94, \mathrm{p}=0.018)$. We observed no difference in the frequencies of the other ACR criteria between the two patients groups.

\section{DISCUSSION}

IL-21 is a cytokine that, upon binding to its receptor (IL-21R), affects the function of a number of immune competent cells (reviewed in Mehta et al). ${ }^{10}$ While IL-21 is produced primarily by activated CD4+ T cells, its receptor is expressed on T, B, NK and dendritic cells, thus accounting for the array of effects IL-21 exerts on these cells. ${ }^{11}{ }^{12} \mathrm{IL}-21 \mathrm{R}$ is associated with the common $\gamma$ cytokine receptor chain, and signals through a number of pathways including Jak1, Jak3, Stat1, Stat3 and Stat5. ${ }^{13}{ }^{14}$

The effects of IL-21 on effector cells are pleomorphic, as IL-21 has been shown to both promote and suppress immune responses. IL-21 has been shown to inhibit the maturation of dendritic cells, and block the LPS-mediated overexpression of CD80, CD86 and MHC class II. ${ }^{12}$ In addition, IL-21 induces apoptosis in primary B cells in vitro. ${ }^{15}$ By contrast, this cytokine promotes B-cell differentiation and plasma cell generation. ${ }^{16} \mathrm{IL}$ 21 effects on B-cell differentiation are at least in part explained by its ability to induce Blimp-1 and Bcl-6 that are involved in plasma cell differentiation and germinal centre formation, respectively. ${ }^{16}$ Furthermore, IL-21 was found to specifically induce the production of IgG1 and IgG3 by human B cells. ${ }^{17}$
Recent studies suggest a possible role for dysregulated IL-21 in autoimmunity. Lupus-prone BXSB-yaa mice have markedly elevated levels of IL-21 in the serum compared to BXSB wildtype mice. ${ }^{16}$ In the sanroque mouse strain, a mutation in the RING-type ubiquitin ligase protein family member, roquin, results in excessive production of IL-21 and a severe lupus-like autoimmune phenotype. ${ }^{18}$ These observations make IL-21 an interesting candidate gene for a role in human SLE.

In this report, we found that SLE is associated with two SNPs in IL-21 using a large sample of SLE patients and healthy controls. Stratification by race reveals this genetic association is found more in European-American than in African-American SLE patients. While we find evidence for association with rs2221903 in African-American SLE patients, there was no association with rs 907715 . This is likely due to a smaller sample size in the African-American patients studied.

The suggestion that SLE patients homozygous for the SLEassociated alleles in IL-21 suffer from less CNS involvement is preliminary and requires replication. A much larger collection of cases and controls is required to establish this association.

More work is needed to identify the actual mutation site in IL-21 that is important in SLE. The genotyped intronal SNPs are likely a surrogate marker for a mutation with functional consequences, rather than being the actual risk mutation. However, our understanding of the significance of non-coding SNPs is evolving. An example is the surprising finding that a synonymous SNP in the Multidrug Resistance 1 gene encoding for P-glycoprotein results in P-glycoprotein with altered drug and inhibitor interactions. ${ }^{19}$ Determining functional consequences of the IL-21 polymorphisms reported herein might improve our understanding of the pathogenesis of SLE and provide new targets for therapeutic interventions.

Acknowledgements: We would like to thank Dr Gary Gilkeson for contributing Gullah samples and Dr Peter K Gregersen for contributing control samples to our studies.

Funding: This publication was made possible by NIH Grant Number P20-RR015577 from the National Center for Research Resources and by funding from the University of Oklahoma College of Medicine (AHS); the NIH (Al24717, Al31584, Al053747, AR12253, AR42460, AR4894, and RR020143), the Alliance for Lupus Research, the Mary Kirkland Fellowship, and the US Department of Veterans Affairs (JBH).

Competing interests: None declared.

\section{REFERENCES}

1. Harley JB, Kelly JA, Kaufman KM. Unraveling the genetics of systemic lupus erythematosus. Springer Semin Immunopathol 2006 Oct;28:119-30.

2. Sawalha $\mathbf{A H}$, Richardson BC. DNA methylation in the pathogenesis of systemic lupus erythematosus. Curr Pharmacogen 2005;3:73-8.

3. Sawalha AH, Harley JB. Antinuclear autoantibodies in systemic lupus erythematosus. Curr Opin Rheumatol 2004;16:534-40.

4. Arbuckle MR, McClain MT, Rubertone MV, Scofield RH, Dennis GJ, James JA, et al. Development of autoantibodies before the clinical onset of systemic lupus erythematosus. N Engl J Med 2003;349:1526-33.

5. Oelke K, Lu Q, Richardson D, Wu A, Deng C, Hanash S, et al. Overexpression of CD70 and overstimulation of IgG synthesis by lupus T cells and T cells treated with DNA methylation inhibitors. Arthritis Rheum 2004;50:1850-60.

6. Ettinger R, Sims GP, Fairhurst AM, Robbins R, da Silva YS, Spolski R, et al. IL-21 induces differentiation of human naive and memory $B$ cells into antibody-secreting plasma cells. J Immunol 2005;175:7867-79. 
7. Tan EM, Cohen AS, Fries JF, Masi AT, McShane DJ, Rothfield NF, et al. The 1982 revised criteria for the classification of systemic lupus erythematosus. Arthritis Rheum 1982;25:1271-7.

8. Gray-McGuire C, Moser KL, Gaffney PM, Kelly J, Yu H, Olson JM, et al. Genome scan of human systemic lupus erythematosus by regression modeling: evidence of linkage and epistasis at 4p16-15.2. Am J Hum Genet 2000;67:1460-9.

9. Barrett JC, Fry B, Maller J, Daly MJ. Haploview: analysis and visualization of LD and haplotype maps. Bioinformatics 2005;21:263-5.

10. Mehta DS, Wurster AL, Grusby MJ. Biology of IL-21 and the IL-21 receptor. Immunol Rev 2004:202:84-95.

11. Parrish-Novak J, Dillon SR, Nelson A, Hammond A, Sprecher C, Gross JA, et al Interleukin 21 and its receptor are involved in NK cell expansion and regulation of lymphocyte function. Nature 2000;408:57-63.

12. Brandt K, Bulfone-Paus S, Foster DC, Ruckert R. Interleukin-21 inhibits dendritic cell activation and maturation. Blood 2003;102:4090-8.

13. Ozaki K, Kikly K, Michalovich D, Young PR, Leonard WJ. Cloning of a type I cytokine receptor most related to the IL-2 receptor beta chain. Proc Natl Acad Sci USA 2000:97:11439-44.
14. Asao H, Okuyama C, Kumaki S, Ishii N, Tsuchiya S, Foster D, et al. Cutting edge: the common gamma-chain is an indispensable subunit of the IL-21 receptor complex. $\mathrm{J}$ Immunol 2001;167:1-5.

15. Mehta DS, Wurster AL, Whitters MJ, Young DA, Collins M, Grusby MJ. IL-21 induces the apoptosis of resting and activated primary B cells. J Immunol 2003; 170:4111-18

16. Ozaki K, Spolski R, Ettinger R, Kim HP, Wang G, Qi CF, et al. Regulation of B cell differentiation and plasma cell generation by IL-21, a novel inducer of Blimp-1 and Bcl-6. J Immunol 2004;173:5361-71.

17. Pene J, Gauchat JF, Lecart S, Drouet E, Guglielmi P, Boulay V, et al. Cutting edge: IL21 is a switch factor for the production of IgG1 and IgG3 by human B cells. J Immunol 2004:172:5154-7.

18. Vinuesa CG, Cook MC, Angelucci C, Athanasopoulos V, Rui L, Hill KM, et al. A RING type ubiquitin ligase family member required to repress follicular helper $\mathrm{T}$ cells and autoimmunity. Nature 2005;435:452-8.

19. Kimchi-Sarfaty C, Oh JM, Kim IW, Sauna ZE, Calcagno AM, Ambudkar SV, et al. A "silent" polymorphism in the MDR1 gene changes substrate specificity. Science 2007;315:525-8. 